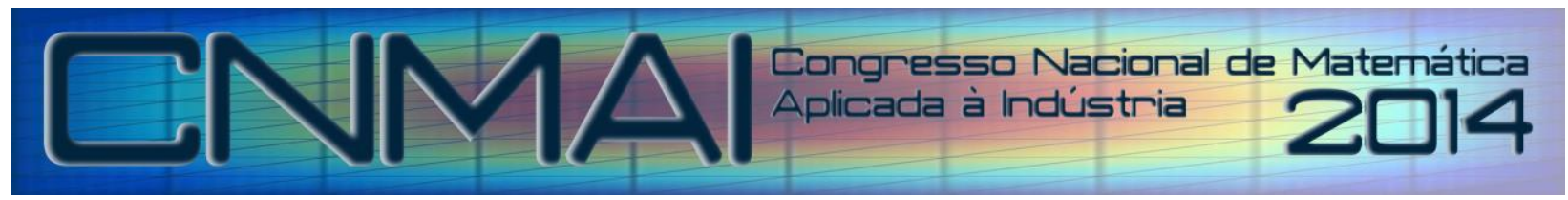

18 a 21 de novembro de 2014, Caldas Novas - Goiás

\title{
ANÁLISE COMPUTACIONAL DE ESFORÇOS ELETROMECÂNICOS NOS ENROLAMENTOS DE TRANSFORMADORES OCASIONADOS POR CORRENTE DE CURTO-CIRCUITO UTILIZANDO O MÉTODO DE ELEMENTOS FINITOS
}

\author{
Diorge de Souza Lima, diorge.lima15@gmail.com¹. \\ Wellington da Silva Fonseca, fonseca@ufpa.br ${ }^{2}$.
}

\begin{abstract}
${ }^{1}$ Universidade Federal do Pará - Campus Universitário de Tucuruí - Faculdade de Engenharia Elétrica - Tucuruí - Pará
${ }^{2}$ Universidade Federal do Pará - Campus de Ananindeua - Faculdade de Ciência \& Tecnologia - Ananindeua - Pará
\end{abstract}

Resumo: Transformadores de potência são equipamentos de grande importância para o sistema elétrico nacional, sendo o responsável pela viabilidade no contexto de transmissão e possibilitando a ligação dos centros geradores aos consumidores. Assim, devido sua importância o projeto desses dispositivos deve ser realizado prevendo situações mais severas a que possa ser submetido. Por outro lado, a prática mostra inúmeras falhas nestes dispositivos, dentre as mais frequentes, destacam-se as causas por esforços eletromecânicos nos enrolamentos ocasionados por estados transitórios. Nessa perspectiva, o presente trabalho tem por objetivo analisar através de método computacional o comportamento de transformadores quando submetidos a corrente de curto-circuito. Para tal analise é utilizado o método de elementos finitos (MEF) para modelagem de um transformador de 50MVA instalado em uma empresa geradora de energia da região Norte. O método utilizado consiste na discretização de um meio contínuo em pequenos elementos mantendo suas características originais, por ser uma forma de resolução numérica de um sistema em equações diferenciais parciais. Para isso, serão apresentadas de maneira detalhada todas as etapas para a obtenção dos resultados, desde o pré-processamento, processamento ao pós-processamento. Por fim, serão apresentadas as análises computacionais e validados com os cálculos apresentados na folha de dados (datasheet) do equipamento.

Palavras-chave: Métodos matemáticos, métodos de elementos finitos, esforços eletromecânicos, transformadores.

\section{INTRODUÇÃO}

A necessidade em otimizar a realização de projetos e análises de transformadores é de extrema importância nas diversas áreas da engenharia elétrica. $\mathrm{O}$ uso de algoritmos eficazes combinado a modelos matemáticos poderosos e principalmente softwares baseados na técnica do método de elementos finitos (MEF) surgiram para atingir tal objetivo. O tempo total do projeto pode ser reduzido significativamente usando-se esse tipo de modelagem, além da possibilidade em criar modelos paramétricos em duas e em três dimensões (SENNA, 2010).

Atualmente, o método de elementos finitos é o mais utilizado para o cálculo numérico de fenômenos eletromagnéticos. Para a sua correta utilização, o objeto de estudo deve ter sua geometria subdividida em várias partes, que serão chamados de elementos finitos. As subdivisões são conhecidas por malhas, sendo normalmente constituídas por triângulos ou quadriláteros em análise bidimensional, ou pirâmides e tetraedros na análise tridimensional. Os vértices dessas malhas são denominados de nó e são utilizados para montar um sistema de equações cuja solução permite determinar as grandezas de interesse no fenômeno analisado. No caso eletromagnético, essa solução é o vetor potencial magnético ou elétrico em cada nó da malha. Assim, com posse dos parâmetros intrínsecos do objeto de estudo, será possível determinar os campos magnéticos e elétricos no interior dos elementos finitos (AHN, 2012).

Nessa perspectiva, utilizou-se um software comercial disponível na versão estudantil baseado no método de elementos finitos para a realização de uma análise eletromagnética em transformadores no estado de curto-circuito. Lima (2014) afirma que esses equipamentos são importantes para a sociedade, pois permitem a ligação dos centros geradores aos consumidores, sendo o grande responsável na redução de perdas na distribuição de energia (FAIZ, 2011).

\section{MÉTODO DE ELEMENTOS FINITOS}

O software utilizado neste trabalho utiliza o MEF para obter os campos em todas as regiões do modelo. Desta forma, a fim de obter as equações a ser resolvida, a geometria do problema é discretizada automaticamente em pequenos elementos, ou seja, todo o modelo sólido é dividido por uma malha de elementos. O conjunto de todos os triângulos é referido como elemento de malha finita. O campo desejado em cada elemento é aproximado por um polinômio quadrático de segunda ordem dado pela Eq. (1) (SENNA, 2010). 


$$
A_{z(x, y)}=a_{0}+a_{1} x+a_{2} y+a_{3} x^{2}+a_{4} x y+a_{5} y^{2}
$$

Dessa maneira, os campos serão calculados para seis pontos, ou seja, os três vértices do triangulo e os pontos médios dos lados destes como na Figura (1a). Já os campos internos ao triangulo são calculados com o uso de uma interpolação quadrática de segunda ordem. A Figura (1b) demonstra esquematicamente a junção das sub-regiões de complexidade menor para a discretização total do problema (SOUZA, 2005).

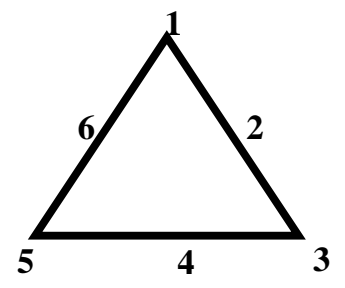

(a)

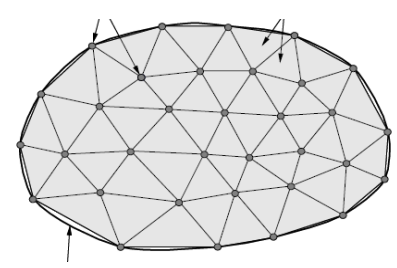

(b)

Figura 1. (a) Pontos de cada elemento utilizados para o calculo dos campos e (b) Malha de elemento Finito (SOUZA, 2005).

Problemas magnetostáticos são problemas em que os campos são invariantes no tempo. A densidade de campo (H) e a densidade de fluxo (B) podem ser calculadas de acordo com a Eq. (2) e a Eq. (3) (KOLONDZOVSKI, 2004).

$$
\begin{aligned}
& \nabla \cdot H=J \\
& \nabla \cdot B=0
\end{aligned}
$$
2010).

Os campos são calculados a partir da Equação de Poisson (4) e substituídos na Eq. (5) da função energia (SENNA,

$$
\begin{aligned}
& \nabla^{2} \cdot A=-\mu \cdot J \\
& F_{(A)}=\frac{1}{2} \int\left(\frac{\nabla A \cdot \nabla A}{J}+A J\right) \partial V
\end{aligned}
$$

Esta função é minimizada com respeito ao valor de A em cada nó de cada triângulo. Mas para todos os triângulos o resultado é um sistema matricial com matrizes esparsas dadas pela Eq. (6). Assim como em todos os métodos, há a presença de um erro, neste caso é devido à aproximação polinomial do vetor potencial magnético. Logo, a Equação de Poisson será da seguinte forma representada pela Eq. (7) (SENNA, 2010).

$$
\begin{gathered}
{[S] \cdot[A]=[J]} \\
\nabla^{2} \cdot A^{\text {aprox }}+\mu \cdot J=R
\end{gathered}
$$

Assumindo que $A$ seja uma função quadrática e R sendo uma constante em cada triângulo da malha, o erro local em cada triângulo será proporcional ao próprio R. Para a análise transitória o software utiliza para solução dinâmica a Eq. (8).

$$
\nabla \times v \nabla \times A=J_{s}-\sigma \frac{\partial A}{\partial t}-\sigma \nabla V+\nabla \times H_{c}+\sigma v \times \nabla \times A
$$

Onde $\nabla$ é o operador vetorial Nabla, $A$ o vetor potencial magnético, $\mu$ a permeabilidade magnética do material, $J$ a densidade de corrente, $R$ o erro local em cada triângulo, $V$ o potencial elétrico escalar, $H_{c}$ o campo magnético permanente, $v$ a velocidade e $\sigma$ a condutividade.

Abaixo será demonstrado na Figura (2) o fluxograma com as etapas contidas no desenvolvimento da análise deste trabalho. Os softwares baseados no método de elementos finitos são estruturados em três etapas, pré-processamento, processamento e pós-processamento. Dessa mesma forma, o pré-processamento deste trabalho consiste no desing da geometria, na escolha da solução e parâmetros do modelo. O processamento será as análises e a convergência dos resultados que serão realizadas pelo software. A etapa final, de pós-processamento, sendo apresentados todos os resultados, logo exige experiência do usuário na correta interpretação dos gráficos apresentados. 


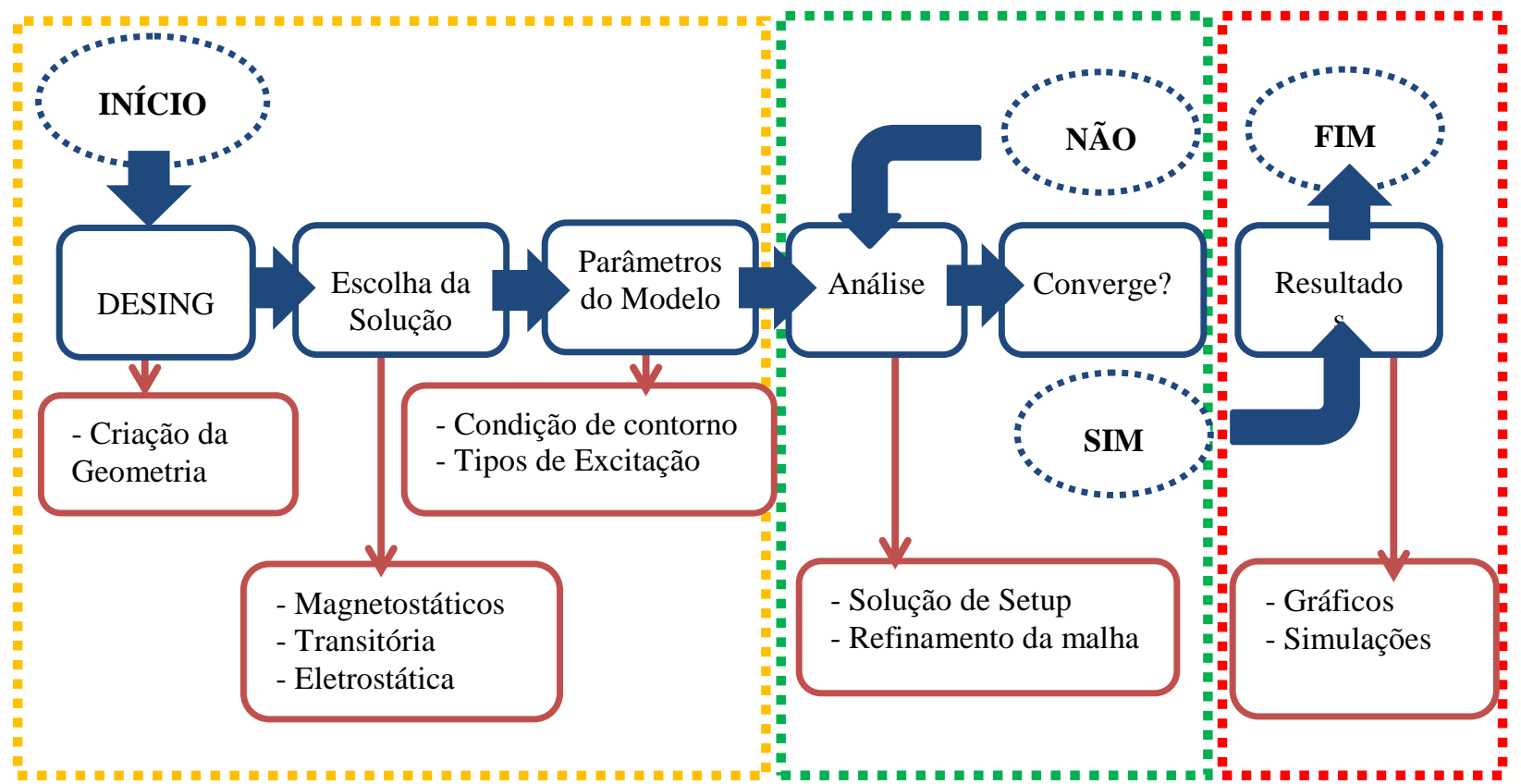

Pré-Processamento

Processamento

Pós-Processamento

Figura 2. Fluxograma do processo de modelagem do software.

\section{CORRENTE DE CURTO-CIRCUITO}

A corrente de curto circuito exercida sobre o enrolamento do transformador é calculada a partir de dados intrínsecos do dispositivo. O potencial magnético vetorial, a densidade do fluxo magnético e a força eletromagnética devido a corrente de curto circuito são resolvidos por análise utilizando o método de elementos finitos baseado no problema eletromagnético. Comumente os transformadores são projetados para resistir ao maior pico da corrente em seus terminais, considerando o equipamento conectado a um barramento infinito do sistema elétrico. Nesta condição, a expressão utilizada para determinar o nível máximo da corrente de curto-circuito no transformador pode ser representada pela Eq. (9) (BRONZEADO, 2007).

$$
I_{c c}=\frac{k \sqrt{2} \cdot S_{n} \cdot 10^{6}}{\sqrt{3} \cdot V \cdot Z}
$$

Sendo $k$ o fator de assimetria da corrente de curto circuito, ${ }_{n}$ a potência nominal do transformador (MVA), $V_{\text {a }}$

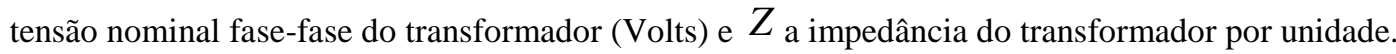

A Figura (3) ilustra a distribuição de fluxo magnético de dispersão entre os enrolamentos de um transformador com bobinas concêntricas, ou seja, as direções das forças eletromagnéticas, correntes e densidade de fluxo magnético (AHN, 2011).

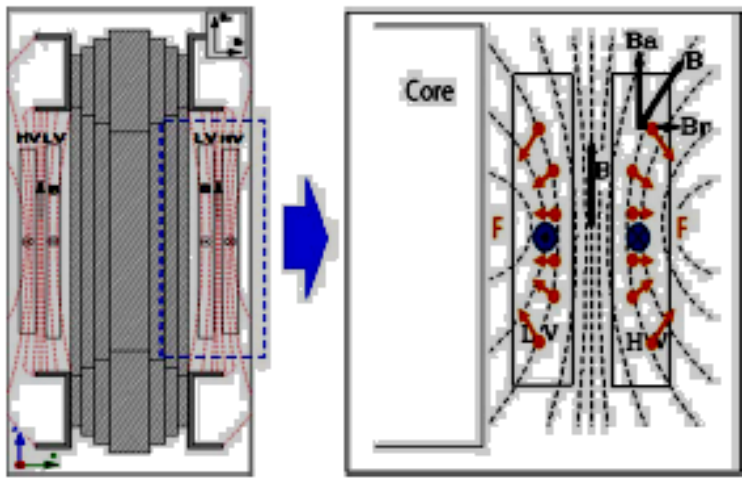

Figura 3. Distribuição do fluxo magnético de dispersão em transformadores com enrolamentos concêntricos (AHN, 2012).

Observa-se que as linhas de fluxo têm uma direção predominantemente axial ao longo da altura dos enrolamentos. No entanto, nas extremidades dos enrolamentos, as linhas de fluxo mudam de direção na tentativa de encurtar o caminho de retorno. Desta forma, o fluxo total de dispersão pode ser decomposto em duas componentes, uma na direção axial e outra na direção radial (LIMA, 2014). 


\section{FORÇAS ELETROMECÂNICAS EM TRANSFORMADORES}

Densidades de forças em um determinado volume de uma bobina são obtidas pela expressão da força de Lorentz, calculada na Eq. (10). Essas forças são geradas pela interação da densidade de corrente e do fluxo magnético de dispersão (BASTOS, 1996). Logo, estão diretamente relacionadas à amplitude de correntes passantes nas bobinas dos transformadores. Para este trabalho, serão realizadas investigações de forças nas bobinas de transformadores na condição de curto-circuito. Onde $\vec{f}$ é a densidade volumétrica de forças $\left(\mathrm{N} / \mathrm{m}^{3}\right)$, $\vec{J}$ é a densidade superficial de corrente $\left(\mathrm{A} / \mathrm{m}^{2}\right)$ e $\vec{B}$ a densidade de fluxo magnético de dispersão (Tesla).

$$
\vec{f}=\vec{J} \times \vec{B}
$$

\subsection{Cálculo das Forças Axiais}

As forças axiais oriundas da componente de fluxo de indução magnética de dispersão radial produzida pela corrente de curto-circuito tendem a comprimir os enrolamentos. Nessa condição a força de compressão atua no enrolamento interno e externo, como demonstrado na Figura (4) (NEVES, 2011 apud LIMA, 2014).

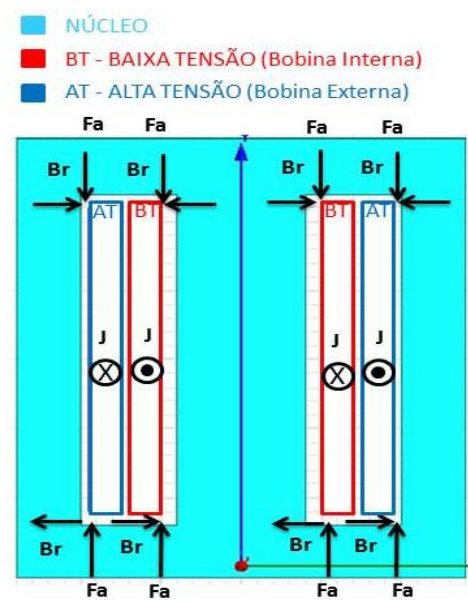

Figura 4. Direção da Força Axial na bobina do transformador na condição de curto-circuito (LIMA, 2014).

As forças axiais serão analisadas como em condição ideal, onde os campos nas duas extremidades das bobinas são direcionados para o ponto médio dos enrolamentos. Na Figura (5) é apresentada de maneira ilustrativa a densidade de fluxo magnético e as forças axiais em ambos os enrolamentos, embora haja uma grande força por unidade de comprimento nas extremidades dos enrolamentos, a força cumulativa de compressão é maior na metade da altura das bobinas nos enrolamentos externos e internos (FONSECA, 2010 apud LIMA, 2014).

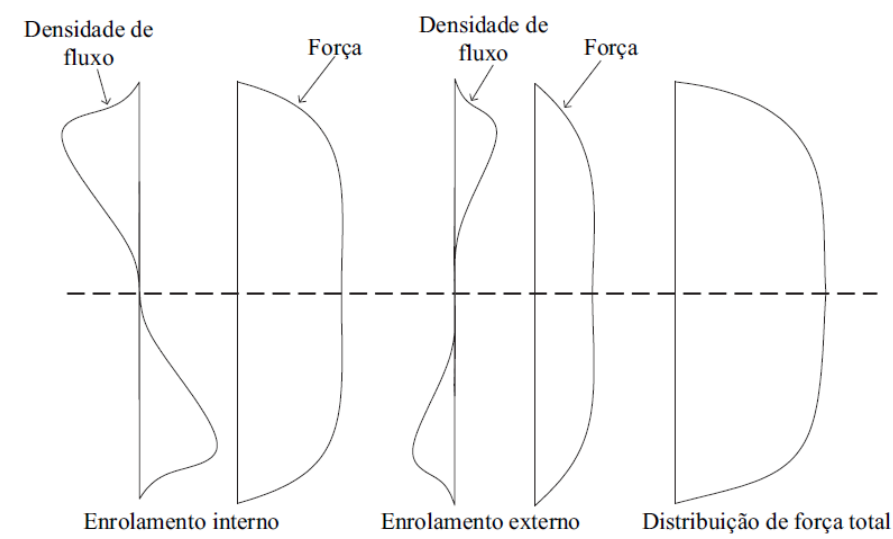

Figura 5. Distribuição do fluxo radial e de força axial em enrolamentos concêntricos iguais (FONSECA, 2010).

Para determinar a força axial pelo método analítico, é necessário conhecer o comprimento efetivo do caminho do fluxo radial $h_{w}$ a densidade de fluxo radial $\mathrm{B}_{\mathrm{r}} \mathrm{e}$ o valor médio dos ampère-espiras, onde $a$ é o comprimento da seção do enrolamento expresso como uma fração do seu comprimento total. A densidade de fluxo radial médio será fornecida pela Eq. (11). A força axial pode ser determinada de acordo com a Eq. (12) (GUIMARÃES, 2008).

$$
B_{r}=\frac{2 \pi \cdot a \cdot(n i)}{10^{4} \cdot h_{w}} \quad[T]
$$




$$
F_{a}=\frac{2 a \cdot(\pi \cdot n \cdot i)^{2}}{h_{w} \cdot 10^{7}} \cdot D_{m} \quad[N]
$$

\subsection{Cálculo das Forças Radiais}

As forças radiais são oriundas da componente de fluxo de indução magnética de dispersão axial e produzem efeitos de compressão nos enrolamentos dos transformadores. Assim, na condição de curto-circuito, a força tende a comprimir o enrolamento interno e expandir o enrolamento externo (WATERS, 1966). Na Figura (6) estão as direções das forças radiais para esta condição (LIMA, 2014).

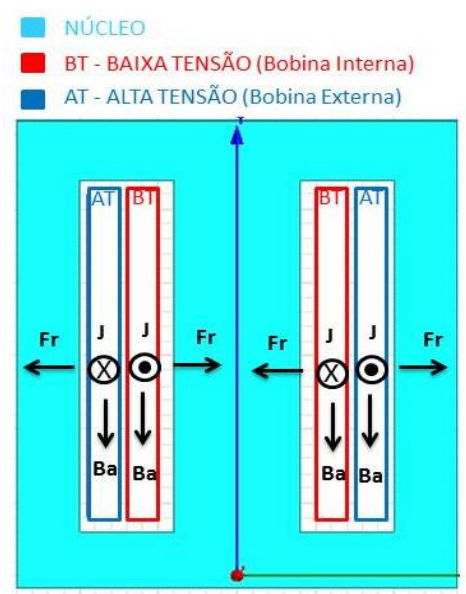

Figura 6. Direção da Força Radial na bobina do transformador na condição de curto-circuito

A densidade de fluxo magnético de dispersão na direção axial, no ponto médio entre os enrolamentos, pode ser determinada pela Eq. (13), onde (ni) representa ampère-volta de cada enrolamento que são responsáveis pela produção de campo axial $\left(\mathrm{B}_{\mathrm{a}}\right)(\mathrm{AZEVEDO}, 2007)$.

$$
B_{a}=\frac{\sqrt{2} \cdot(n i) \cdot 4 \pi \cdot 10^{-7}}{h}
$$

Desprezando a redução da componente axial do campo que interage nas extremidades da bobina e do campo de fluxo, é gerada uma força radial média. Dessa forma, as forças radiais atuando em cada enrolamento com diâmetro médio $D_{m}$ e altura $h$ podem ser determinadas pela Eq. (14) (AZEVEDO, 2007).

$$
F_{\text {rmed }}=\frac{2 \pi \cdot(n \cdot i)^{2}}{h \cdot 10^{7}} \cdot D_{m} \quad[N]
$$

\section{MODELAGEM DE UM TRANSFORMADOR}

O modelo utilizado é um transformador monofásico de 50MVA com o núcleo de ferro silício do tipo envolvido. O mesmo é utilizado por uma empresa geradora de energia localizada na região Norte do país. Para a criação do modelo foram adotadas as medidas e dimensionamentos iguais ao projeto do dispositivo. A Tabela (1) apresenta as principais características desse dispositivo.

Tabela 1. Características dos enrolamentos do transformador de 50MVA.

\begin{tabular}{|c|c|c|}
\hline \multirow{2}{*}{ Características } & \multicolumn{2}{|c|}{ Enrolamento } \\
\cline { 2 - 3 } & Externo & Interno \\
\hline Diâmetro Interno $(\mathrm{mm})$ & 1406 & 1096 \\
\hline Diâmetro Externo $(\mathrm{mm})$ & 1599 & 1262 \\
\hline Altura Axial $(\mathrm{mm})$ & 2080 & 2080 \\
\hline Altura Radial $(\mathrm{mm})$ & 96 & 83 \\
\hline Número de Espiras & 572 & 191 \\
\hline Frequência $(\mathrm{Hz})$ & 60 & 60 \\
\hline Potência $(M V A)$ & 50 & 50 \\
\hline Tensão de Fase $(k V)$ & 132,80 & 39,84 \\
\hline Corrente de Fase $(A)$ & 376,55 & 1255,12 \\
\hline Ligação & Estrela & Estrela \\
\hline
\end{tabular}




\subsection{Construção do Núcleo e Bobinas}

Para as simulações e análise em transformadores apresentadas neste trabalho foram utilizados modelos matemáticos. Com isso, inicialmente criou-se a geometria seguindo a riscas todas as características de projeto do dispositivo, as medidas utilizadas estão demonstradas na Tabela (1).

\subsection{Definição dos Materiais}

Com a construção do núcleo e das bobinas, deve-se associar um material a estes elementos. O software possui uma série de materiais pré-definidos, além de permitir a inserção de novos materiais definidos pelo usuário por uma tabela de valores. Para este trabalho, utilizou-se para o núcleo o material aço-silício (Steel 1010) e para as bobinas o Cobre (Cooper). Os procedimentos são simples, basta selecionar a geometria, por conseguinte selecionar o material específico de cada parte do equipamento.

\subsection{Definição da Excitação}

Assim como toda máquina elétrica, o transformador também deve ser inicialmente excitado com as características desejadas de operação. A excitação desse dispositivo ocorrerá através dos terminais criados nas bobinas. Para isso, deve-se indicar o número de voltas e o respectivo sentido dos condutores. O enrolamento criado pode ser alimentado de três formas distintas: por corrente, por tensão ou por circuito externo.

\subsection{Condição de Contorno}

Assim como em qualquer problema eletromagnético criado em softwares baseados no método de elementos finitos deve-se criar uma região fechada em torno do problema, com intuito de isolar a região onde se deseja calcular os fluxos existentes. Neste trabalho, existem vários tipos de regiões que podem ser criados pelo software, porém será utilizado um isolamento simples, ou seja, que não haja influência externa.

\subsection{Verificação dos Resultados}

Nesta etapa do trabalho é considerada importante quanto às demais. Pois cabe ao projetista, a partir dos conhecimentos adquiridos ao longo de sua carreira, reconhecer e interpretar os resultados demonstrados pelo programa. As simulações foram realizadas em um transformador monofásico de 50MVA utilizado por uma empresa de geração de energia, então com alguns valores disponíveis no datasheet do equipamento, será possível validar a utilização deste software como uma ferramenta eficaz nas análises de sistemas eletromagnéticos.

\section{RESULTADOS DA SIMULAÇÃO}

Este tópico destina-se a apresentar e discutir os principais resultados oriundos das simulações computacionais dos transformadores de potência submetidos a estado de curto-circuito e em operação nominal. Dessa maneira, inicialmente serão demonstrados resultados obtidos de simulações de um transformador monofásico de 50MVA em operação normal. As simulações para esta condição operativa foram executadas utilizando correntes monofásicas calculadas de acordo com os dados disponibilizados na placa do equipamento, sendo valor nominal de 376,55A no enrolamento externo e 1255,12A no enrolamento interno.
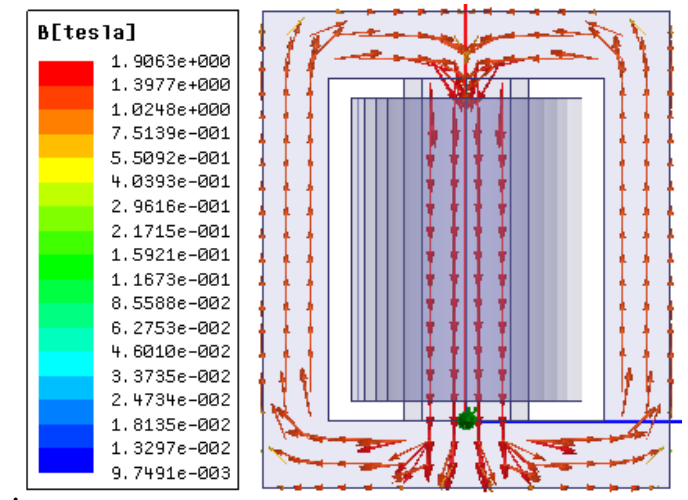

(a)

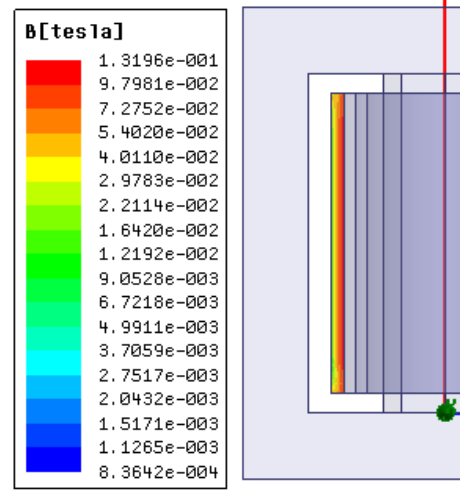

(b)

Figura 7. Densidade de fluxo magnético de dispersão no (a) núcleo e no (b) enrolamento do transformador em condição normal.

Para a simulação no estado de curto-circuito utilizou uma corrente igual a 8979A no enrolamento externo e 34586A no enrolamento interno, todos os valores foram calculados de acordo com a Equação (9) com o fator de assimetria igual a 2,69. A Figura (8) ilustra a densidade de fluxo magnético do transformador durante o curto circuito. Assim como esperado, a distribuição de fluxo magnético no interior do transformador sofre alteração no caminho preferencial, passando parte do fluxo a circular pelo ar. 

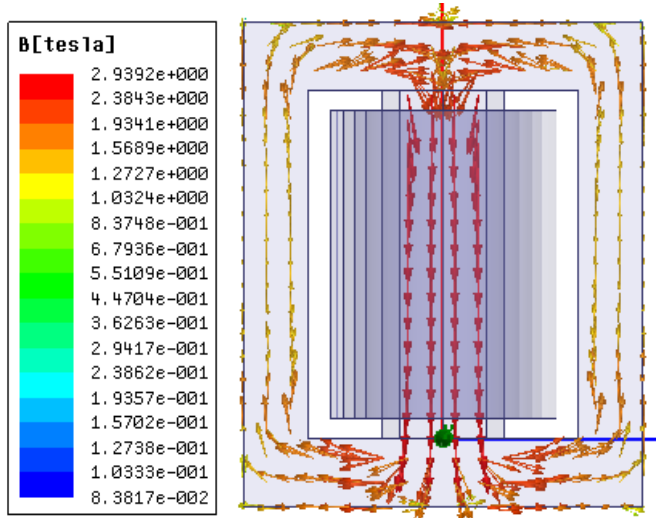

(a)
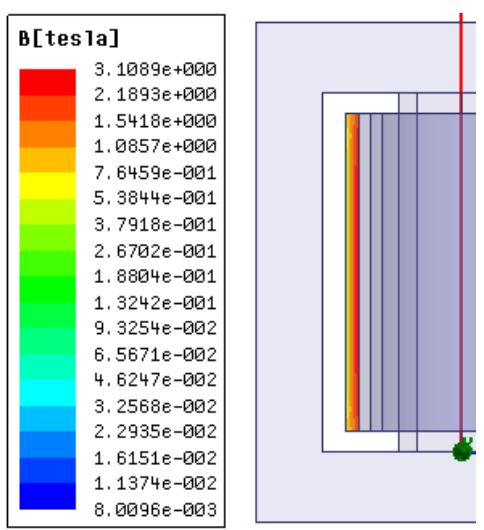

(b)

Figura 8. Densidade de fluxo magnético de dispersão no (a) núcleo e no (b) enrolamento do transformador em curto-circuito.

Para entender melhor os resultados, abaixo será demostrado a diferença do comportamento do fluxo magnético no enrolamento do transformador em diferentes situações, sendo estas em operação normal e em curto-circuito. Na Figura (9) (a) é possível observar que a densidade de fluxo magnético de dispersão, assim como esperado, é praticamente constante ao longo da altura do enrolamento, sofrendo variação nas extremidades superiores e inferiores. Para esta condição operacional, o valor de pico encontrado nas simulações são próximos a $125 \mathrm{mT}$ na região central dos enrolamentos. De maneira similar, na Figura (9) (b) a densidade de fluxo tem o valor de pico próximo a 2,93 T.

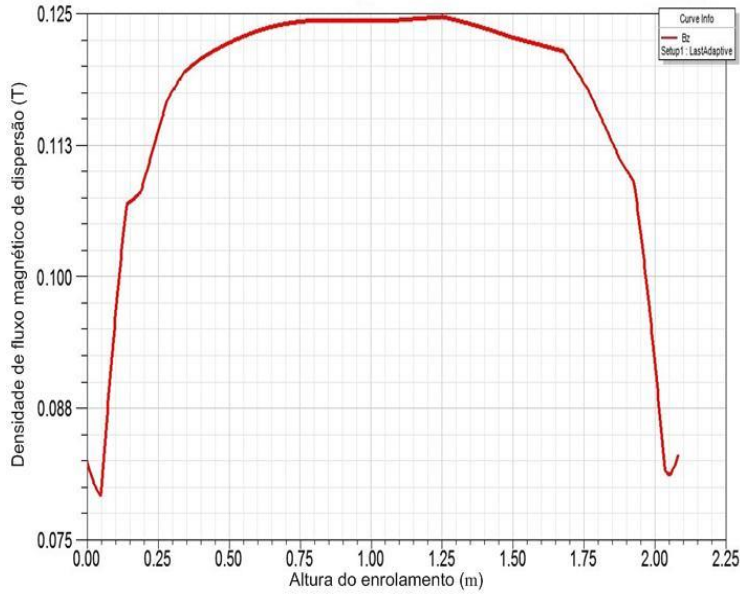

(a)

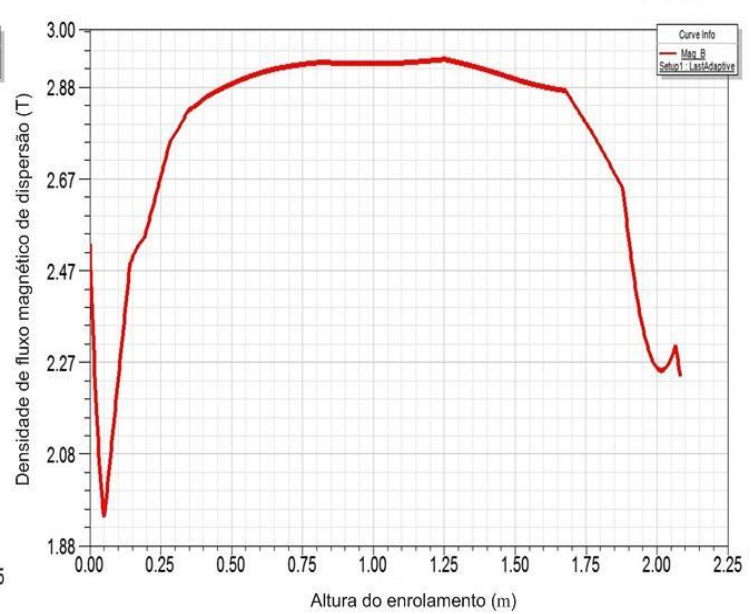

(b)

Figura 9. Densidade de fluxo magnético de dispersão em função da altura do enrolamento em (a) condição nominal e em (b) curto-circuito.

Logo a partir da comparação desses resultados é possível observa o efetivo aumento na amplitude na densidade de fluxo magnético de dispersão. Isso corrobora efetivamente no aumento excessivo das forças exercidas nos enrolamentos dos transformadores. Para a melhor compreensão desses valores montou-se a Tabela (2) com intuito de compara a densidade de fluxo magnético de dispersão axial na condição nominal com a condição de curto-circuito.

Tabela 2. Comparação dos valores da densidade de fluxo magnético de dispersão axial para a condição nominal e de curto-circuito.

\begin{tabular}{|c|c|c|}
\hline $\begin{array}{c}\text { Densidade de fluxo magnético de } \\
\text { dispersão axial [T] }\end{array}$ & Condição Nominal & Curto-circuito \\
\hline Resultado Analítico & $2,42 \cdot 10^{5}$ & $1,14 \cdot 10^{8}$ \\
\hline Resultado Computacional & $2,22 \cdot 10^{5}$ & $1,62 \cdot 10^{8}$ \\
\hline
\end{tabular}

A Figura (10) apresenta a força axial distribuída em função da altura do enrolamento interno para o transformador monofásico de 50MVA na condição nominal de operação e de curto-circuito. Como esperado tem-se efeito de compressão no enrolamento. As maiores forças estão localizadas nas extremidades. 


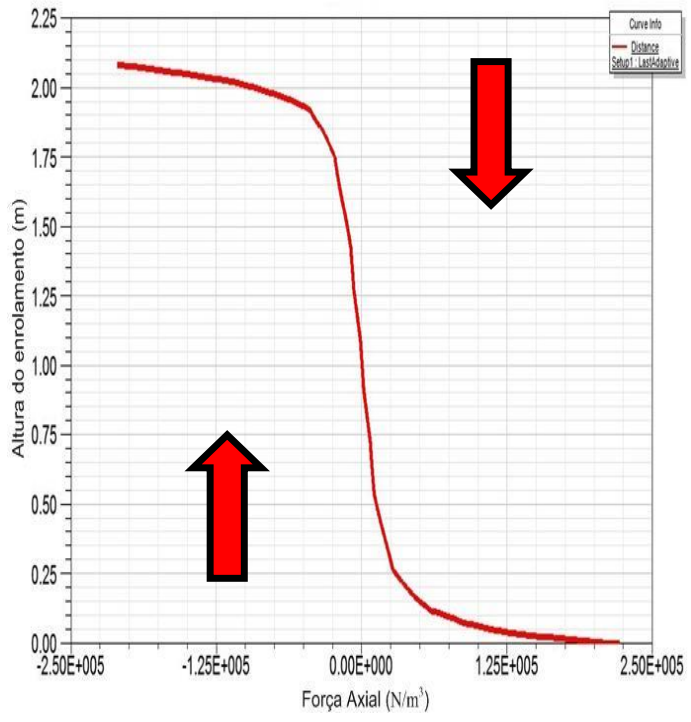

(a) Condição nominal

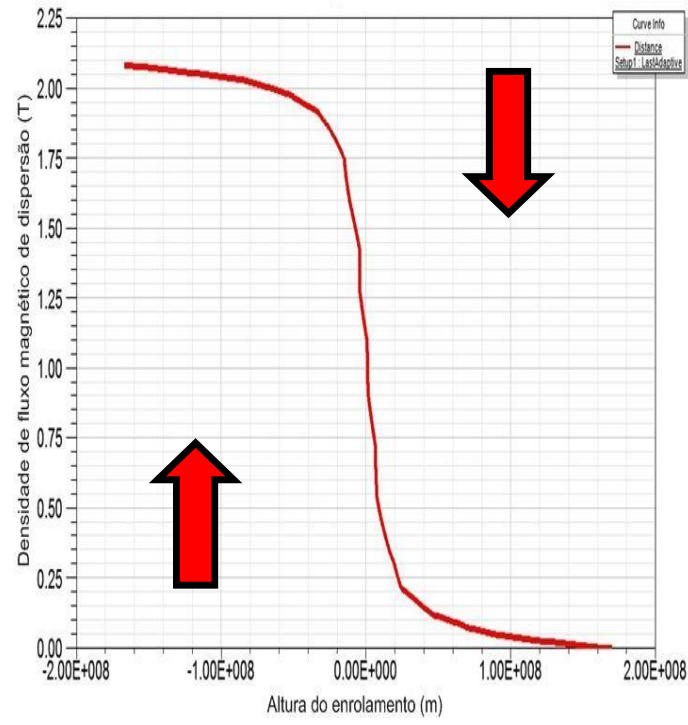

(b) Curto-circuito

Figura 10. Comparação da força axial distribuída no enrolamento interno do transformador de 50MVA.

Os valores axiais nas extremidades podem ser comparados com aqueles obtidos pela metodologia analítica apresentados na Tabela (3).

Tabela 3. Comparação entre simulação e metodologia analítica da força axial na condição nominal e em curto circuito.

\begin{tabular}{|c|c|c|}
\hline Força Axial $\left[\mathbf{N} / \mathbf{m}^{3}\right]$ & Condição Nominal & Curto-circuito \\
\hline Resultado Analítico & $2,42 \cdot 10^{5}$ & $1,14 \cdot 10^{8}$ \\
\hline Resultado Computacional & $2,22 \cdot 10^{5}$ & $1,62 \cdot 10^{8}$ \\
\hline
\end{tabular}

A Figura (11) apresenta a força radial distribuída em função da altura do enrolamento interno em condição nominal de operação.
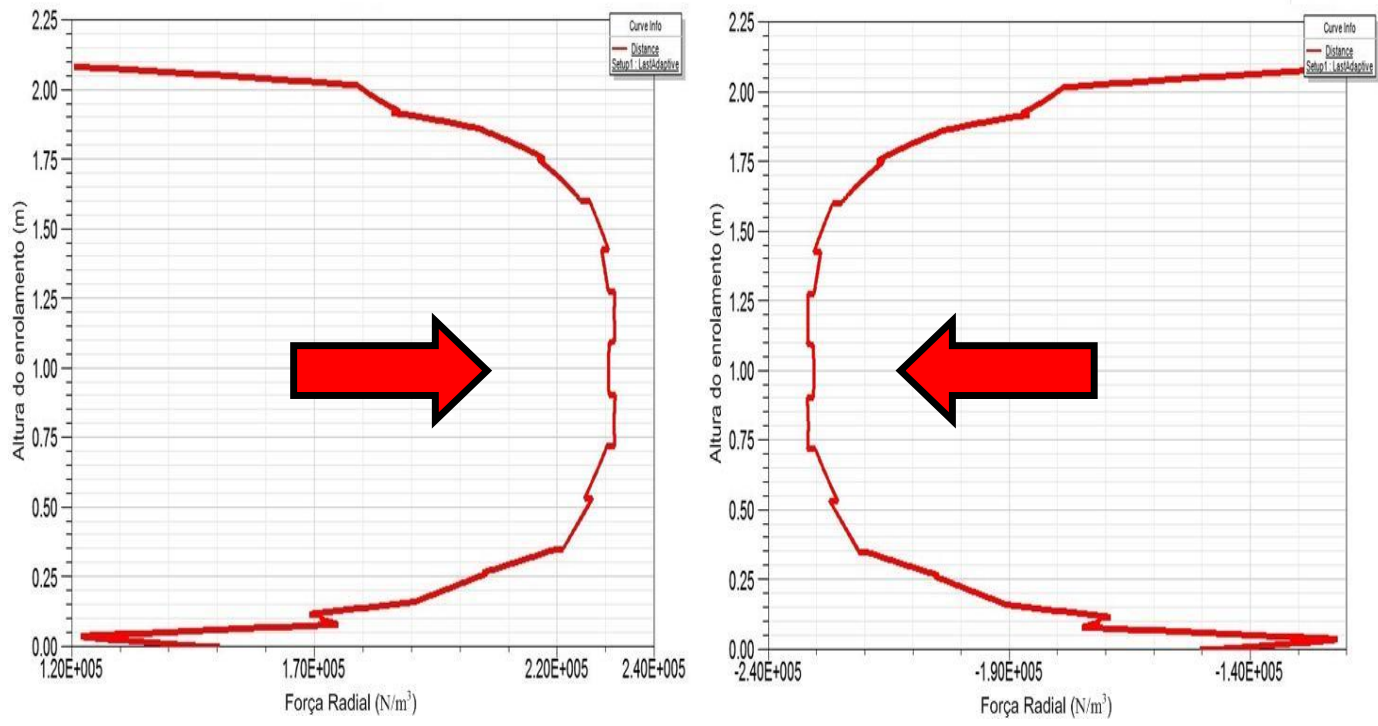

Figura 11. Força radial de compressão distribuído no enrolamento interno do transformador de 50MVA em condição nominal.

A Figura (12) apresenta a força radial distribuída em função da altura do enrolamento interno em condição nominal de operação. 

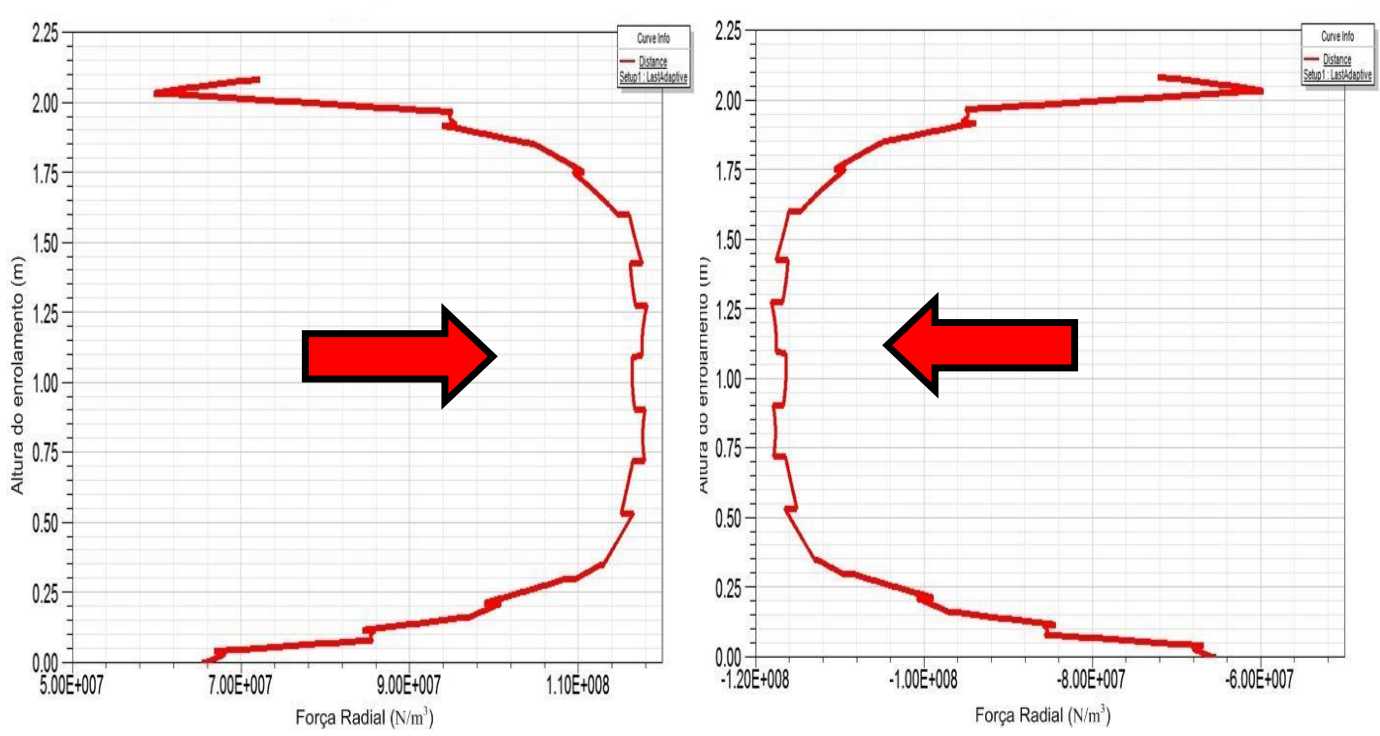

Figura 12. Força radial de compressão distribuído no enrolamento interno do transformador de 50MVA em condição de curto-circuito.

Os valores radiais dos enrolamentos internos em condição nominal e em curto-circuito são observados pelas Figuras (11) e (12), respectivamente. Sendo apresentam amplitudes diferentes de acordo com a condição de operação do equipamento. Por isso, esses valores podem ser comparados com aqueles obtidos pela metodologia analítica sendo apresentados na Tabela (3).

Tabela 4. Comparação entre simulação e metodologia analítica da força radial na condição nominal e em curto circuito.

\begin{tabular}{|c|c|c|}
\hline Força Axial [N/m³] & Condição Nominal & Curto-circuito \\
\hline Resultado Analítico & $2,33 \cdot 10^{6}$ & $1,12 \cdot 10^{8}$ \\
\hline Resultado Computacional & $2,32 \cdot 10^{6}$ & $1,17 \cdot 10^{8}$ \\
\hline
\end{tabular}

A partir dos valores demonstrados nas Tabelas (3) e (4), é possível observar que ao submeter o transformador a estados transitórios de curto-circuito, as forças axiais e radiais aumentam consideravelmente. Assim, é valido ressaltar que sob as condições de distúrbio este equipamento pode sofrer danos devido às elevadas amplitudes de forças.

\section{CONSIDERAÇÕES FINAIS}

As simulações no software foram realizadas para dois casos distintos. Inicialmente foi analisado o transformador em condição nominal, onde se pode visualizar a densidade de campo magnético produzida no núcleo do transformador. A magnitude da densidade magnética obtidas nas simulações está de acordo com o esperado. Além disso, nota-se que em situação nominal de operação, o fluxo de dispersão nos enrolamentos é bem reduzido. Desta forma, associado com a baixa corrente do enrolamento, os esforços serão de menores intensidades.

O segundo caso foi um estudo com o transformador submetido a um curto-circuito. A distribuição da densidade de fluxo magnético para está condição foi diferentemente da situação nominal citada anteriormente em que a distribuição da densidade de fluxo magnético no interior do transformador sofre alterações no caminho preferencial pelo núcleo magnético e passando a circular parte do fluxo pelo ar. Também se verificou um elevado valor do fluxo de dispersão nos enrolamentos, logo consequentemente os esforços são de maiores intensidades, que foi possível observar nos resultados demonstrados neste trabalho.

Portanto, ressalta-se que o deste trabalho foi estabelecer uma metodologia computacional confiável, que possa oferecer sustentação aos estudos de desempenho dos equipamentos sob investigação em um programa que utilizada método de elementos finitos para as suas análises.

\section{REFERÊNCIAS}

Ahn, H. M.; Lee, J. Y.; Kim, J. K.; Jung, S. Y.; Oh, Y. H.; Hahn, S. C. "Finite element analysis of short circuit electromagnetic force in power transformer.” IEEE Trans. Ind. Appl., Vol. 47, No. 3, pp. 1267-1272, Mar. 2011. 
Ahn, H. M.; Lee, J. Y.; Kim, J. K.; Jung, S. Y.; Oh, Y. H.; Hahn, S. C. "Experimental Verification and Finite Element Analysis of Short-Circuit Electromagnetic Force for Dry-Type Transformer," IEEE Transactions on Magnetics, Vol. 48, No. 2, Feb. 2012.

Azevedo, A. C. "Estresse Eletromecânico em Transformadores Causados por Curtos-circuitos passantes e correntes de Energização". 111p. Tese (Doutorado) - Universidade Federal de Uberlândia, Uberlândia, Brasil, 2007.

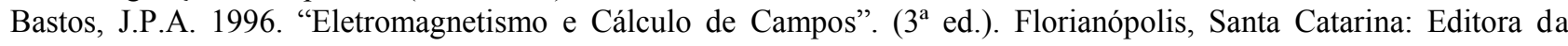
UFSC.

Bronzeado, H.S., Azevedo, A. C., Delaiba, A. C., Oliveira, J. C., Carvalho, B. C. "Estresse Eletromecânico em Transformadores: Análise no Domínio do Tempo". IV Congresso de Inovação Tecnológica em Energia Elétrica CITENEL, Araxá, Brasil, 07p, 2007.

Faiz, J., Ebrahimi, B.M., Elhaija, W.A., "Computation of Static and Dynamic Axial and Radial Forces on Power Transformer Windings Due to Inrush and Short Circuit Currents". IEEE Jordan Conference on Applied Electrical Engineering and Computing Technologies (AEECT), 08p, 2011.

Fonseca, W.S., Lopes, A.C., Nunes, M.V. "Modelagem de Esforços Eletromecânicos de Transformador de Núcleo Amorfo através do Método de Elementos Finitos". Anais do T\&D Transmition and Distribuition IEEE Latino América, São Paulo, Brasil, 06p, 2010.

Guimarães, R., Delaiba, A.C., Saraiva, E., Rosentino Jr, A.J.P., Chaves, M.L.R., Bronzeado, H.S., Silva, C.A. "Investigação sobre Estresses Eletromecânicos em Transformadores causados pela corrente de Energização (Inrush)". 8th IEEE/IAS International Conference on Industry Applications - INDUSCON, Poços de Caldas, Brasil, 08p, 2008.

Kolondzovski, Z. "Evaluation of Methods for Calculation Synchronous Generator Reactances" International PhDseminar Computation of Electromagnetic Fields, 23-28 September 2004, Budva / Serbia \& Montenegro

Lima, D. S., Fonseca, W. S., Furtado, M. S., Lima, A. K. F. "Análise de Esforços nos Enrolamentos de Transformadores Ocasionados por Correntes de Energização Utilizando o Método de Elementos Finitos” CONEM - Congresso Nacional de Engenharia Mecânica. Uberlândia - MG, Brasil, 08p, 2014.

Neves, W., Fernandes Jr, D., Baltar, F. J. A., Rosentino Jr, A.J.P., Saraiva, E., Delaiba, A.C., Guimarães, R., Chaves, M.L.R., Oliveira, J.C. "A Comparative Investigation of Electromechanical Stresses on Transformers Caused by Inrush and Short-Circuit Currents". 11th International Conference - Electrical Power Quality and Utilization EPQU, Lisboa, Portugal, 06p, 2011.

Senna, L. E. "Relatório de Estágio Supervisionado". Santa Maria - RS, 2010. Disponível em: < http://pt.scribd.com/doc/129193256/Relatorio-de-estagio-Luciano-Senna-2010>. Acesso em 08 de abril de 2014.

Souza, R. M. “O Método dos Elementos Finitos Aplicados ao Problema de Condução de Calor". Belém - PA, 2003. Disponível em: < http://www.inf.ufes.br/ luciac/fem/livros-fem/ApostilaElementosFinitosNiCAE.pdf> Acesso em: 02 de Fevereiro de 2014.

Waters, M. 1996. "The Short-Circuit Strength of Power Transformers”. McDonald \& Co. Ltd, London.

\title{
COMPUTATIONAL ANALYSIS OF STRESS ELETROMECHANICAL IN WINDING TRANSFORMERS DUE BY CURRENT SHORT CIRCUIT USING FINITE ELEMENT METHOD
}

\author{
Diorge de Souza Lima, diorge.lima15@gmail.com¹. \\ Wellington da Silva Fonseca, fonseca@ufpa.br ${ }^{2}$.
}

${ }^{1}$ Universidade Federal do Pará - Campus Universitário de Tucuruí - Faculdade de Engenharia Elétrica - Tucuruí - Pará

${ }^{2}$ Universidade Federal do Pará - Campus de Ananindeua - Faculdade de Ciência \& Tecnologia - Ananindeua - Pará

\begin{abstract}
Power transformers are very important equipment for the national electric system, being responsible for viability in the context of transmission and enabling the connection of generators to the consumer centers. Thus, because of its importance the design of these devices must be performed by providing for more severe situations that may be submitted. On the other hand, practice shows numerous failures in these devices, among the most frequent causes stand out efforts by electromechanical windings caused by transient states. In this perspective, the present work aims to analyze through computational method's behavior when subjected to current transformer shorting. For this analysis the finite element method (MEF) modeling of a 50MVA transformer installed in a power generating company in the northern region is used. The method consists of the discretization of a continuous medium in small elements retaining its original character, being a form of numerical solution of a system of partial differential equations. To do so, will be presented in detail all the steps to obtaining the results from the pre-processing, processing, post-processing. Finally, computational analysis will be presented and validated with the calculations presented in the datasheet (datasheet) equipment.
\end{abstract}

Keywords: Mathematic method, finite method elements, stress electromagnetics, transformers. 